\title{
artigo
}

Martins da Silva, D.K.M.; Santos, F.A.; Duarte, R.B.; Batista e Silva, D.P.; Soares, D.G.; Cabral, R.L.;

Os desafios da enfermagem como coordenadora do cuidado frente à pandemia da COVID-19

\section{Os desafios da enfermagem como coordenadora do cuidado frente à pandemia da COVID-19}

\author{
The challenges of nursing as a care coordinator in the face of the COVID-19 pandemic
}

Los retos de la enfermería como coordinadora de los cuidados en la pandemia del COVID-19

\begin{abstract}
RESUMO
Objetivo: Descrever a experiência de enfermeiros que atuam na linha de frente ao combate da COVID-19 nos cenários do interior e capital do estado do Ceará. Método: Trata-se de um estudo descritivo, com abordagem qualitativa, tipo relato de experiência, elaborado a partir das experiências vivenciadas por enfermeiros que atuam na linha de frente no combate a COVID-19 no contexto da Atenção Primária à Saúde (APS). Tal relato apresenta como recorte temporal, as atividades desenvolvidas pelas enfermeiras durante o período de março a junho de 2020. A coleta das informações para a escrita do relato se deu no mês de julho de 2020. Resultados: À vista desse contexto, é possível selecionar os resultados em duas dimensões: nas unidades na qual os autores deste trabalho vivenciam a Pandemia: Fluxos operantes dos serviços e Desafios e potencialidades vivenciadas. Conclusão: Vale destacar que os enfermeiros deste estudo estão na linha de frente, demonstrando seu compromisso no enfrentamento da COVID-19.
\end{abstract}

DESCRITORES: Pandemia; Coronavírus; Infecções por Coronavírus; Enfermagem.

\section{ABSTRACT}

Objective: To describe the experience of nurses who work in the front line to combat COVID-19 in the countryside and capital scenarios of the state of Ceará. Método: Trata-se de um estudo descritivo, com abordagem qualitativa, tipo de relato de experiência, elaborado a partir das experiências vivenciadas por enfermeiros que atuam na linha de frente ao combate a COVID-19 no contexto da Atenção Primária à Saúde (APS). This report presents as a temporal cut, the activities developed by nurses during the period from March to June 2020. The collection of information for the writing of the report took place in the month of July 2020. Results: In view of this context, it is possible to select the results in two dimensions: in the units in which the authors of this work experience the Pandemic: Services operating flows and Challenges and potentialities experienced. Conclusion: It is worth noting that the nurses in this study are on the front line, demonstrating their commitment to confronting COVID-19.

DESCRIPTORS: Pandemic; Coronavirus; Coronavirus infections; Nursing.

\section{RESUMEN}

Objetivo: Describir la experiencia de las enfermeras que trabajan en la primera línea de combate al COVID-19 en los escenarios del campo y de la capital del estado de Ceará. Método: Se trata de un estudio descriptivo, con enfoque cualitativo, tipo informe de experiencia, elaborado a partir de las vivencias de las enfermeras que trabajan en primera línea para combatir el COVID-19 en el contexto de la Atención Primaria de Salud (APS). Este informe presenta en forma de corte de tiempo, las actividades desarrolladas por las enfermeras durante el periodo de marzo a junio de 2020. La recogida de información para la redacción del informe tuvo lugar en julio de 2020. Resultados: A la vista de este contexto, es posible seleccionar los resultados en dos dimensiones: en las unidades en las que los autores de este trabajo viven la pandemia: Flujos operativos de los servicios y Desafíos y potencialidades vividas. Conclusión: Cabe destacar que las enfermeras de este estudio están en primera línea, demostrando su compromiso a la hora de enfrentarse al COVID-19.

DESCRIPTORES: Pandemia; Coronavírus; Infecciones por coronavirus; Enfermería.

RECEBIDO EM: 30/01/2021 APROVADO EM: 16/02/2021

\section{Daniele Keuly Martins da Silva}

Enfermeira pelo Centro Universitário Fametro (UNIFAMETRO). Especialista em Saúde Coletiva pelo Centro Universitário Fametro (UNIFAMETRO). Mestranda do Programa de Pós-Graduação em Saúde Coletiva (PPSAC) da Universidade Estadual do Ceará (UECE). Bolsista da Fundação Cearense de Apoio ao Desenvolvimento Cientifico e Tecnológico (FUNCAP).

ORCID: 0000-0002-5026-5176 


\section{Francisca Antonia dos Santos}

Enfermeira pelo Centro Universitário Fametro (UNIFAMETRO). Enfermeita Assistencial na Atenção Primaria a Saúde no Município de General Sampaio.

ORCID: 0000-0002-2834-4126

\section{Rafael Bezerra Duarte}

Enfermeiro. Docente do curso de Bacharelado em Enfermagem no Centro Universitário Vale do Salgado. Mestrando em Saúde Coletiva pela Universidade Estadual do Ceará.

ORCID: 0000-0002-2280-0864

\section{Débora Pena Batista e Silva}

Enfermeira (UECE). Mestranda no Programa de Pós-Graduação em Saúde Coletiva (UECE).

ORCID: 0000-0003-0633-5242

\section{Delane Giffoni Soares}

Enfermeira (UECE). Egressa da Residência Multiprofissional em Saúde da Família e Comunidade pela Escola de Saúde Pública do Ceará (ESP-CE). Especialista em Saúde da Família e Comunidade. Mestranda no Programa de Pós-Graduação em Saúde Coletiva (PPSAC) pela Universidade Estadual do Ceará. Bolsista da Coordenação de Aperfeiçoamento de Pessoal de Nível Superior (CAPES).

ORCID: 0000-0002-1215-5291

\section{Riksberg Leite Cabral}

Enfermeiro (UECE). Mestre em Saúde da Família - UECE Docente do Centro Universitário FAMETRO Técnico de Auditoria de Gestão do Núcleo de Contas Médicas do Hospital Municipal Dr. João Elísio de Holanda Tutor do Curso de Aperfeiçoamento em Gerência de UBS - UFF e DAB/MS.

ORCID: 0000-0002-0795-5569

\section{INTRODUÇÃO}

D enominado SARS-CoV-2, o novo Coronavírus, causador da doença COVID-19, foi detectado em 31 de dezembro de 2019 em Wuhan, na China. A Organização Mundial da Saúde (OMS) confirmou a circulação do novo Coronavírus em 9 de janeiro de $2020^{(1)}$. Portanto, declarou a doença como uma Emergência de Saúde Pública de importância internacional, sendo considerada uma pandemia a partir do mês de março, devido aos vários surtos ocorridos em diversos países do mundo ${ }^{(2)}$.

No Brasil, o Ministério da Saúde (MS) confirmou o primeiro caso da doença em 26 de fevereiro. Após essa data, a velocidade de propagação se deu a níveis alarmantes de contaminação em todo o País ${ }^{(3)}$. Com o aparecimento do novo vírus tornou-se um desafio frente à estrutura de vigilância existente no território nacional, sobretudo no momento em que a redução de investimentos do Sistema Único de Saúde (SUS) e na pesquisa debilita a capacidade de detecção precoce e de resposta à doença $a^{(4)}$.
Segundo o último boletim epidemiológico do Estado do Ceará, publicado no início de fevereiro de 2021, é observada uma crescente no número de casos, com uma taxa de mortalidade acumulada no ano de 2020 e 2021 de 116,2 por 100 mil habitantes. Ainda neste mesmo documento, refere que no ano atual a taxa de mortalidade está em 3,6 óbitos por 100 mil habitantes, com destaque para algumas regiões do interior que apresentaram grandes incrementos quando comparados com semanas anteriores ${ }^{(5)}$. Com o impacto causado desde o início pela pandemia, mudanças abruptas nas rotinas das instituições de saúde foram ocasionadas e junto a isso o processo de adaptação dos profissionais de saúde diante do novo cenário de crise sanitária.

Nesse contexto, a enfermagem se configura como uma profissão em destaque por atuarem na linha de frente contra a COVID-19, prestando seus cuidados de forma contínua, enfrentando condições ineficazes de trabalho, sem suporte que garantam sua proteção individual e, portanto, trazendo reflexões sobre a sua impor- tância enquanto membro de uma equipe de saúde que trabalha em prol de prevenir, restabelecer e salvar vidas ${ }^{(6)}$.

As práticas que compreendem o processo de trabalho dos profissionais de enfermagem, assim como a necessidade de reestruturação da Atenção Primária à Saúde trazem à tona os diversos desafios que precisam ser vistos como prioridade e que são tratados com descaso pelo Governo Federal. Com essa conjuntura atual, ficou evidenciada a importância de se ter sistemas públicos de saúde robustos, com capacidade abrangente de atender as diversas demandas existentes, independente do cenário instaurado e a relevância do SUS e dos seus trabalhadores da saúde para a sustentabilidade do Estado, na garantia de prover o bem-estar social para a população como um todo ${ }^{(7)}$.

Assim, o objetivo deste estudo foi descrever a experiência de enfermeiros que atuam na linha de frente ao combate da COVID-19 nos cenários do interior e capital do estado do Ceará. Esta pesquisa é relevante para profissionais e acadêmicos da área de saúde, assim como para a socie- 


\section{artigo}

dade em geral. Justifica-se, pois, a compreensão do trabalho desempenhado pelos enfermeiros, auxilia no delineamento de estratégias de enfrentamento dessa crise sanitária ocasionada pelo coronavírus nos contextos individual e coletivo.

\section{MÉTODOS}

Trata-se de um estudo descritivo, com abordagem qualitativa, tipo relato de experiência, elaborado a partir das experiências vivenciadas por enfermeiros que atuam na linha de frente ao combate a COVID-19 no contexto da Atenção Primária à Saúde (APS). Tal relato apresenta como recorte temporal, as atividades desenvolvidas pelas enfermeiras durante o período de março a junho de 2020. A coleta das informações para a escrita do relato se deu no mês de julho de 2020.

Utilizou-se como critérios de inclusão, os relatos, as observações e as experiências vivenciadas pelas enfermeiras diante de suas atuações e atividades desenvolvidas, sendo levadas em consideração a gerência da assistência, de pessoas, materiais e fluxos frente à pandemia da COVID-19, bem como suas vivências associadas com o estresse, a pressão e os desafios de lidar com o ofício, acrescido do risco de adoecer.

As atividades foram vivenciadas em uma Unidade de Atendimento Básico (UAB), pertencente ao município de Maracanaú, Ceará, e em uma Unidade Básica de Saúde (UBS), pertencente ao município de General Sampaio, este considerado geograficamente no interior, também do Estado do Ceará.

A UAB foi inaugurada em abril de 2018 e tem funcionamento 24 horas. A unidade dispõe de atendimentos somente a pacientes residentes no município e funciona como retaguarda. Está situada no complexo do Hospital Municipal Dr. João Elísio de Holanda com equipes médicas e de enfermagem sendo voltada para pacientes de baixo risco, ou seja, a mesma assume atendimento de menor gravidade. Este serviço apresentou-se como referência para o tratamento da COVID-19.

Já a UBS possui somente uma equipe de enfermagem que atua prestando atendimento ambulatorial, serviços de consultas de enfermagem dentro dos programas da atenção primária e vigilância em saúde aos pacientes de baixo risco. Este serviço apresentou-se como referência para tratamento de COVID-19 por meio do acolhimento de sintomáticos, busca ativa dos contactantes e monitoramento dos mesmos. Deste modo, a mesma ainda realiza o acolhimento e triagem primária, e direcionam estes pacientes dentro da rede de atenção à saúde, encaminhando-os para avaliações médicas e realização do teste rápido para a COVID-19.

Os dados aqui relatados traduzem a vivência presencial, os quais emergiram de relatos, observações, estudos e discussões entre as profissionais na estrutura interna das instituições onde eram desenvolvidas as atividades de trabalho dos mesmos. Sendo o presente estudo um relato de experiência, não foi necessária a utilização do Termo de Consentimento Livre e Esclarecido (TCLE), assim como envio para aprovação de um Comitê de Ética e Pesquisa (CEP).

\section{RESULTADOS}

À vista desse contexto pandêmico, é possível selecionar os resultados em duas dimensões. Primeiramente, os Fluxos operantes dos serviços, que compreendem o momento em que as unidades foram referenciadas à população como porta de entrada para o combate ao coronavírus. Ainda, fora decidido pelas coordenações dos serviços de saúde que os trabalhadores pertencentes ao grupo de risco, idosos e pessoas com doenças crônicas, deveriam ser afastados ou realocados em outros setores da instituição. Aqueles que apresentaram sintomas respiratórios foram afastados e testados para COVID-19.

As instituições deste estudo, disponibilizaram uma área exclusiva para o atendimento destes casos, prestando o atendimento separadamente dos demais pacientes. As mesmas adotaram medidas de isolamento com a finalidade de prestação de uma assistência isolada destes casos, redimensionando as equipes disponíveis para $\mathrm{o}$ atendimento.

A segunda dimensão se refere aos Desafios e potencialidades vivenciadas, eixo que traz uma reflexão considerando o aumento do consumo de Equipamentos de Proteção Individual (EPI) com a epidemia da COVID-19. Deste modo, uma grande preocupação da equipe de enfermagem era com a necessidade de racionalização e a possibilidade da falta destes materiais nas unidades. Além da intensa rotina, na qual os profissionais foram expostos à apreensão destes por não possuírem total domínio quanto ao uso adequado dos EPI's, tornava-se mais desafiador a atuação para toda a equipe.

As unidades deste relato não realizaram discussões necessárias entre os trabalhadores da saúde a respeito de medidas que melhorem as condições de trabalho durante este período, bem como capacitaçôes para fortalecer o conhecimento dos perigos e aquisição de comportamento seguro frente à pandemia da COVID-19, o que tornou este período ainda mais desafiador, trazendo repercussões físicas e psíquicas para estes profissionais.

\section{DISCUSSÃO}

A OMS definiu 2020 como o ano dos profissionais de enfermagem, o que seria uma celebração global(8). Porém, a classe trabalhadora perpassa por diversos cenários desafiadores diante dessa conjuntura de pandemia da COVID-19.

Com a chegada deste cenário pandêmico ao nosso cotidiano assistencial nas unidades básicas de saúde, uma das maiores preocupações dos profissionais enfermeiros concentrava-se em como realizar um atendimento aos pacientes que adentravam aos serviços como sendo casos suspeitos ou confirmados para a COVID-19(9).

Contudo, é conhecido que a $\mathrm{CO}$ VID-19, tornou-se um dos maiores desafios dentro das práticas de saúde, uma vez que, a mesma possui alto potencial de contágio rápido, e também, considerada letal. Sem protocolos definidos para o controle e o combate deste vírus, acaba por gerar 
dúvidas e apreensões, principalmente devido às mudanças recorrentes nos fluxos de atendimentos ${ }^{(10)}$.

Com isso, os protocolos institucionais para atendimentos de casos suspeitos e confirmados para COVID-19, encontram-se em constantes mudanças, o que gera desafios maiores para o enfermeiro no direcionamento de suas ações assistenciais $^{(11)}$. Até a conclusão do presente relato, o fluxo se dava através de uma triagem rápida realizada por um enfermeiro e, após isso, o mesmo era encaminhado de acordo com a sua gravidade sintomática, podendo este ser direcionado ao tratamento e isolamento domiciliar. Sendo assim, era acompanhado pela equipe de saúde da família ou prosseguia para a rede secundária com maior suporte assistencial ao mesmo.

Diante do contexto epidemiológico atual, o manejo clínico na atenção primária deve ser realizado de forma minuciosa, com avaliação precisa dos sintomas, visto que a APS é a porta de entrada do SUS e tem papel fundamental na resposta global à doença, ofertando um atendimento resolutivo e com total capacidade de identificação precoce de casos graves que devem ser encaminhados ao serviço especializado ${ }^{(12)}$.

Dentro desta perspectiva, torna-se imprescindível uma normatização de fluxos padrões dentro da Rede de Atenção à Saúde (RAS) para que se possa determinar um atendimento uniformizado e organizado em todas as instituições de saúde ${ }^{(13)}$.

O MS ainda recomenda o fluxo rápido, otimizando o atendimento no momento da chegada e estabelecendo um nível de prioridade dos mesmos, tendo a disposição um médico, um enfermeiro e um técnico de enfermagem para a assistência, assim como a área exclusiva também é tida como uma recomendação absoluta e indispensável ${ }^{(14)}$.

Desta forma, o serviço de saúde possui o dever de fornecer capacitação para todos os profissionais de saúde para a prevenção da transmissão de agentes infecciosos. Todos os profissionais de saúde devem ser treinados para o uso correto e seguro dos $\mathrm{EPI}^{(15)}$.
Alerta-se diante desta pandemia, para a saúde mental dos profissionais de enfermagem, que passam a correr risco aumentado para desenvolvimento da síndrome de Burnout, já que o medo, insegurança e apreensão eram prevalentes entre estes com o avanço da doença. Recentemente, a OMS, publicou um guia para orientar cuidados à saúde mental de diversos grupos, incluindo profissionais de saúde. Para os trabalhadores da saúde, lidar com o seu trabalho, acrescido do risco de adoecer, provocam severos problemas de saúde mental ${ }^{(16)}$.

O distanciamento dos familiares, o medo e o estresse devido a mudança na rotina de forma inesperada, onde a categoria é colocada à prova frente à uma crise sanitária grande, acaba impactando em consequências físicas, emocionais e psicológicas diante do desafio diário de lidar com números significativos de infectados e mortes pela doença ${ }^{(17)}$.

Por sua vez, o trabalho da equipe de enfermagem requer competência técnica e científica, conhecimento, habilidade e controle emocional sobre a prática, tendo em vista que, a assistência apresenta situações de risco, desgaste físico e emocional, responsabilidades com a vida das pessoas e enfrentamento de medos e sofrimentos. Toda essa situação impacta negativamente na satisfação com o trabalho, resultando em prejuízos na assistência, qualidade do cuidado e segurança do paciente ${ }^{(18)}$.

Entre os profissionais de enfermagem, em consonância com a literatura, os fatores desencadeantes da depressão podem estar relacionados ao processo de trabalho, como o turno, o relacionamento entre profissional-paciente, profissional-família e profissional-profissional, a sobrecarga de trabalho, o desgaste, o suporte social, o conflito de interesses e as estratégias de enfrentamento desenvolvidas ${ }^{(19-20)}$.

A pressão de lidar com o ofício e o risco de adoecimento fortalece um estado de vulnerabilidade psíquica entre os profissionais de enfermagem atuantes nas unidades estudadas, além das preocupações econômicas e trabalhistas relacionadas às recomendações de distanciamento social $^{(21)}$. Soma-se ainda a insegurança decorrente das frequentes atualizaçôes nos fluxos de atendimento e protocolos de saúde os quais interrompem e alteram a rotina de trabalho das equipes de saúde.

O profissional de Enfermagem sempre trabalhou sem condições de exercer seu melhor papel. Salários defasados, estruturas que não ofertam condições mínimas de trabalho, como por exemplo, a baixa disponibilidade de materiais de proteção ${ }^{(22)}$. A falta de EPI's são situações corriqueiras dentro dos nossos processos de trabalho. Desta forma, a população deve ficar ciente que a COVID-19, não trará facilidade para a Enfermagem, porém, assim como nas outras epidemias vivenciadas em seus cotidianos assistenciais, a categoria está disposta a enfrentar essa tempestade com técnica, ciência e profissionalismo.

\section{CONCLUSÃO}

O presente relato evidenciou as experiências de enfermeiros atuantes na Atenção Primária à saúde no contexto da pandemia da COVID-19 em duas vertentes que concernem a padrões regionais no estado do Ceará. O seu desenvolvimento permitiu compreender que, mesmo em momentos de comoção, a equipe de enfermagem (re)inventou ações para garantir a atenção à saúde aos usuários.

Tendo em vista que o vírus foi recentemente descoberto e que ainda seguem em desenvolvimento os estudos sobre o tema visto a sua magnitude, os protocolos e recomendações sofrem mudanças constantes. Vale destacar que os enfermeiros deste estudo estão na linha de frente, demonstrando seu compromisso no enfrentamento da COVID-19. Deste modo, enfatiza-se, a relevância da manutenção de contínua atualização técnico-científica para a utilização adequada das barreiras à exposição, assim como os ajustes na estrutura dos fluxos operacionais dos serviços.

Portanto, almeja-se estimular a socialização de experiências e salientar a necessidade do reconhecimento dos profissionais de enfermagem, uma vez que estes possuem grandes cargas de tra- 


\section{artigo}

Martins da Silva, D.K.M.; Santos, F.A.; Duarte, R.B.; Batista e Silva, D.P.; Soares, D.G.; Cabral, R.L.;

Os desafios da enfermagem como coordenadora do cuidado frente à pandemia da COVID-19

balho, remunerações não condizentes e, ainda assim, assumem os desafios de estar na linha de frente no combate ao novo coronavírus. Portanto, é importante o reconhecimento da complexidade do trabalho do Enfermeiro e de toda equipe de enfermagem, uma vez que possuem como militância o cuidado e manutenção da saúde das populações. -

\section{REFERÊNCIAS}

1. World Health Organization. IHR procedures concerning public health emergencies of international concern (PHEIC). https:// www.who.int/ihr/procedures/pheic/en/ [internet]. 2020 [acesso em 12 jun 20]. Disponivel em: https://www.who.int/ihr/procedures/pheic/en/

2. Organização Pan-Americana da Saúde (OPAS). Folha informativa COVID-19 - Escritório da OPAS e da OMS no Brasil. 2021 [acesso em 12 fev. 2021]. Disponível em: https://www.paho.org/ $\mathrm{pt} /$ covid19

3. Ministério da Saúde (BR). Ministério da Saúde confirmou o primeiro caso de coronavírus em São Paulo. [internet]. Brasília-DF. 2020 [acesso em 12 jun 20]. Disponível em: https://www.saude. gov.br/noticias/agencia-saude/46435-brasil-confirma-primeiro-caso-de-novo-coronavirus

4. Lana RM, Coelho FC, Gomes MFC, Cruz OG, Bastos LS, Villela DAM, et al. Emergência do novo coronavírus (SARS-CoV-2) e o papel de uma vigilância nacional em saúde oportuna e efetiva. Caderno de Saúde Pública. 2020; 36 (3)13.

5. Governo do Estado do Ceará. Secretaria da Saúde. Boletim Epidemiológico. Doença pelo novo Coronavírus (COVID-19). 2021; $\mathrm{n}^{\circ} 04$ [acesso em 12 fevereiro 2021]. Disponível em: https:// www.saude.ce.gov.br/wp-content/uploads/sites/9/2020/02/ BOLETIM_COVID-19_N4_04_02_21_V02.pdf

6. Oliveira AC. Challenges faced by nursing professionals in the COVID-19 pandemic. REME- Rev. Min. Enferm. 2020 [cited february 12,2021$] ; 24: e-1302$. Available from: https://cdn.publisher.gn1.link/reme.org.br/pdf/en_e1302.pdf.

7. Souza IL, Oliveira SA, Rafael RMS, Rodrigues PHA. A ATENÇÃO PRIMÁRIA À SAÚDE NA PÓS-PANDEMIA E A PRÁTICA DE PROFISSIONAIS DE ENFERMAGEM III. Enfermagem na atenção básica no contexto da COVID-19 / Brasília, DF : ABen/DEAB, 2020. 87 p.

8. OPAS/OMS BRASIL. OMS define 2020 como ano internacional dos profissionais de enfermagem e obstetrícia. [Internet]. Disponivel em: https://www.paho.org/bra/index. php?option=com_content $\&$ view $=$ article $\&$ id $=6092:$ oms - de fine-2020-como-ano-internacional-dos-profissionais-de-enfermagem-e-obstetricia\&Itemid=844. 2020.

9. Teodosio SSCS, Gadelha MJA, Alcântara MS, Correia MLR, Freitas MLFO, Dantas RB. O ENFRENTAMENTO DA COVID-19 NA ATENÇÃO PRIMÁRIA EM SAÚDE: UMA EXPERIÊNCIA EM NATAL-RN VII. Enfermagem na atenção básica no contexto da COVID-19 / Brasília, DF: ABen/DEAB, 2020. 87p.

10. Word Health Organization. Considerations for quarantine of individuals in the context of containment for coronavirus disease (COVID-19): Interim guidance [Internet]. Geneva (CH); 2020 [cited 2020 Mar 24]. Available from: https://apps.who.int/iris/handle/10665/331299
11. Rodrigues NH, Silva LGA. Gestão da pandemia Coronavírus em um hospital: relato de experiência profissional. J. nurs. health. 2020. v.10. n. esp. ed. 20104004.

12. Ministério da Saúde (BR). Secretária de atenção primária à saúde. Protocolo de manejo clínico do Coronavírus (Covid-19) na atenção primária à saúde: versão 6 / Coronavirus (Covid-19) clinical management protocol in primary health care: version 6. [internet]. Brasília-DF. 2020 [acesso em 21 jun. 20]. Disponível em: https://pesquisa.bvsalud.org/portal/resource/pt/ biblio-1087335

13. Santos ER, Sicsú NA, Oliveira LB, Silva AS, Ferreira DS, Lima KJV. A ENFERMAGEM EM ATENÇÃO PRIMÁRIA À SAUDE NO CONTEXTO DA PANDEMIA DE COVID-19 NA REGIÃO AMAZÔNICA VI. Enfermagem na atenção básica no contexto da COVID-19 / Brasília, DF: ABen/DEAB, 2020. 87p.

14. Ministério da Saúde (BR). 2 ${ }^{a}$ Etapa Fluxogramas COVID-19 SAES Z [Internet]. Brasília; 2020. [acesso em 2020 abr 20]. Disponivel em: https://www.saude.gov.br/images/pdf/2020/marco/20/2-EtapaFluxogramas-COVID-19-SAES-Z.pdf

15. Agência Nacional de Vigilância Sanitária (ANVISA). Orientações para serviços de saúde: medidas de prevenção e controle que devem ser adotadas durante a assistência aos casos suspeitos ou confirmados de infecção pelo novo Coronavírus (SARSCOV-2). [Internet]. 2020.

16. World Health Organization (WHO). Mental health and psychosocial considerations during the COVID-19 outbreak [Internet]. 2020[cited 2020 Apr 20]. Available from: https://www.who. int/docs/defaultsource/coronaviruse/mental-healthconsiderations.pdf?sfvrsn=6d3578af_2

17. Jackson D, Bradbury-Jones C, Baptiste D, Gelling L, Morin K, Neville $S$ et al. Life in the pandemic: some reflections on nursing in the context of COVID-19. J Clin Nurs. 2020 [cited 2020 Jun 04];6. Available from: https://doi.org/10.1111/jocn.15257.

18. Vieira NF, Nogueira-da-Terra FS. Avaliação do estresse entre os enfermeiros hospitalares. Rev Enferm UERJ. 2017; 25 (14)53.

19. Trettene AS, Costa RB, Prado PC, Tabaquim MLM, Razera APR. Estresse: realidade vivenciada por enfermeiros atuantes em um Centro de Terapia Intensiva. Rev Enferm UERJ. 2018;26(17)52-3.

20. Silva DSD, Tavares NVS, Alexandre ARG. Depression and risk of suicide in professional Nursing: integrative review. Rev Esc Enferm USP. 2015;49(6):1027-36.

21. Campos, D.M.S.; Costa, E.N.F.; Branco, F.M.;Relato de experiência da vivência de residentes de enfermagem frente a pandemia COVID-19. SaúdeColetiva (10) N.59: 4184-4188. 2020.

22. Domingues PHS, Faustino AM, Cuz KCT. A enfermagem em destaque na pandemia da COVID-19: uma análise em mídias sociais. Enferm. Foco 2020; 11 (Esp. 2): 97-102.] 